\title{
Chitosan Loses Innate Beneficial Properties after Being Dissolved in Acetic Acid: Supported by Detailed Molecular Modeling
}

Ismail Bilican, Sami Pekdemir, M. Serdar Onses, Lalehan Akyuz, Ergin Murat Altuner, Behlul Koc-Bilican, Lian-Sheng Zang, Muhammad Mujtaba, Povilas Mulerčikas, and Murat Kaya*

Cite This: ACS Sustainable Chem. Eng. 2020, 8, 18083-18093

Read Online

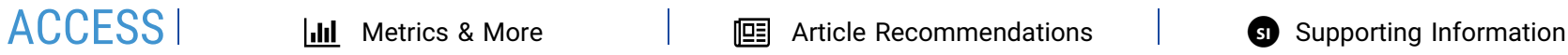

ABSTRACT: Chitosan, which is obtained via deacetylation of chitin, has a variety of uses in agriculture, food, medicine, pharmaceuticals, and cosmetics. Industrial chitosan is in a gel form, which is produced by dissolving in acetic acids. These gels can be chitosan-only films or composite films that include other ingredients such as plant extracts or other polymers. Chitosanbased films, however, are not as natural as chitosan dissolved in weak acids, and they lack some of chitosan's innate properties. In this study, natural chitosan films (NCFs) were obtained from the pupa shells of black soldier flies through a process that maintains the original structure. The semisynthetic film (SCF) was then produced by dissolving the same NCF in acetic acid along with

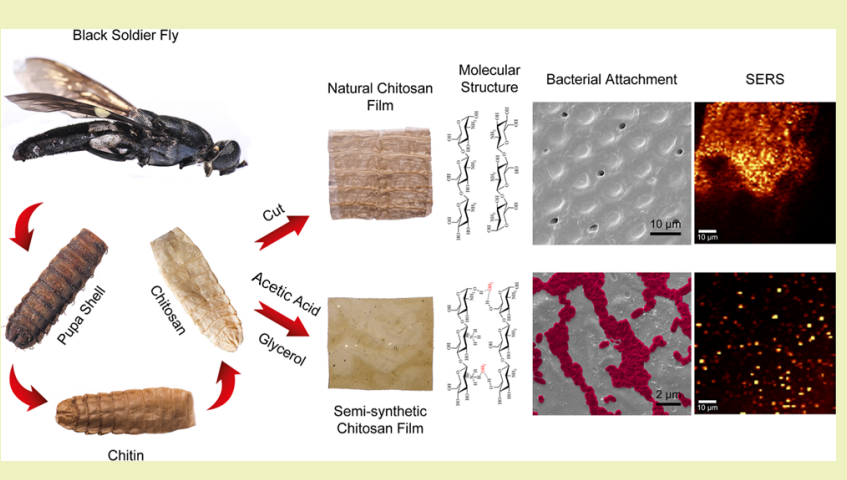
glycerol and glutaraldehyde. The semisynthetic film remarkably lost the beneficial properties of the natural film. The deteriorated characteristics include hydrophobicity, crystallinity, thermal properties, as well as a loss of fibril structure and a reduction in bacterial attachment. Moreover, the Ag-deposited NCFs manifested strikingly higher surface-enhanced Raman scattering activity as compared with the semisynthetic ones. These results, including the molecular modeling data, demonstrate that dissolving chitosan in acetic acid changes its polymeric structure.

KEYWORDS: chitosan film, natural, synthetic, black soldier fly, SERS

\section{INTRODUCTION}

Chitosan is produced by the deacetylation of chitin, which is the second most abundant polymer in the world and is widely used in the film technology. Chitosan-based films are produced by dissolving chitosan powder in acetic acid, and the solution is then plasticized with the addition of a plasticizer such as glycerol. ${ }^{1}$ The dissolution of chitosan in acetic acid at the nontoxic concentration $(<1 \%)$ enables easy formation of films. Chitosan films have numerous industrial applications including, but not limited to, food coating, agriculture, pharmacy, and biomedicine. ${ }^{2,3}$ Furthermore, it is an attractive material for a multitude of applications as it can be easily shaped by molding. The biodegradable, biocompatible, antimicrobial, and antioxidant nature of chitosan makes this material more useful for film applications. ${ }^{2}$ Moreover, thanks to its predispositions to chemical modifications due to its functional chemical structure, chitosan facilitates composite film formation by mixing with different kinds of polymers, plant extracts, essential oils, and proteins to increase these desired properties. ${ }^{4-8}$ The composite formation with other matrices or biomolecules is driven by a series of chemical events, which leads to significant modifications in the overall polymeric chemistry of chitosan. The alteration of the chemical properties of chitosan during film formation affects its physicochemical characteristics.
However, there is no information in the literature on how the physicochemical and biological properties of chitosan powder used in these studies change vis-à-vis films obtained after dissolving in acetic acid.

Commercially available chitosan samples, and samples used in previous studies, are in powder, granule, or flake form. ${ }^{9}$ In a limited number of recent studies, chitin isolation has been carried out by preserving the $3 \mathrm{D}$ structure of the organisms. $^{10,11}$ However, until now, chitosan has not been produced by preserving the $3 \mathrm{D}$ structure of an organism. For this reason, there seems to be no comparison available on the properties of chitosan films produced synthetically by dissolving chitosan in acetic acid, with natural chitosan film (NCF), which is produced by preserving its three-dimensional structure directly from the organism. There is also a need for molecular modeling studies to shed light on the differences between natural and semisynthetic films. Previous efforts

Received: August 28, 2020

Revised: November 14, 2020

Published: December 4, 2020 
focused on the structural differences between $\alpha$ and $\beta$-chitin with chitosan using molecular dynamics simulation methods in aqueous solutions. ${ }^{12}$ However, the conformational flexibility of chitin and chitosan was investigated using Monte Carlo simulations in terms of the freedom of the glycosidic bond by Svokstrup et al. ${ }^{13}$ Although rheological properties of chitosan have been investigated using a viscometric function in acetic acid, the quantum computational methods have not been used for investigation of the stability of chitosan in an acetic acid solution. Therefore, the present study aims to understand the solvent effect on the stability of NCFs and semisynthetic chitosan films (SCFs).

The black soldier fly (BSF), Hermetia illucens (Diptera: Stratiomyidae), is rapidly receiving growing attention in recent years due to its value as an environmental and resource insects. Though originally native to the Americas, it is widely distributed in tropical and temperate regions throughout the world. $^{14,15}$ There are many studies on BSF for waste management and its high nutrient potential. ${ }^{16-18}$ Furthermore, it is reported that BSF is a chitin source. ${ }^{19}$ In a study, chitin was isolated for the first time from a BSF and showed to have an $\alpha$ form. ${ }^{20}$ In another study, the isolation of chitin was made in the waste shells remaining after the pupa stage and it was emphasized that this structure was rich in chitin content. ${ }^{21}$ In all of these studies, chitins were isolated in the powder form from different life stages of the BSF. Until this time, chitin and chitosan production has never been made by keeping the $3 \mathrm{D}$ structure intact from the shell of a BSF pupa, which remained completely waste when they turned into an adult. The BSF, which produces an economically significant amount of larvae, also creates waste shells. ${ }^{20}$ In this study, NCFs were produced from the shells that emerged as waste from the life cycle of a BSF, for the first time. The SCF was produced by adding plasticizer and glutaraldehyde after dissolving the same chitosan in acetic acid. It is aimed to show the physiochemical difference between the NCF and SCF, how the antimicrobial property changes according to surface morphologies, surface changes by SERS analysis, changes in the polymer structure and degradability properties. Accordingly, the following questions will be answered in this study by using quantum chemical calculations: (i) what are the thermodynamically stable configurations for the optimum configurations of chitosan-dimer (CD), CD-acetic acid complex (CDA), aqueous $\mathrm{CD}$ ( $\mathrm{ACD})$, and aqueous protonated CD (PACD); and (ii) how does the stability of $C D$ change when experimental conditions are simulated taking into account the conditions of aqueous acetic acid solution?

\section{MATERIALS AND METHODS}

Sample Collection and Materials. BSF pupa shells were collected from a culture in Vytautas Magnus University, Lithuania in May 2019. $100 \mathrm{~g}$ of BSF pupa shells (around 11,000 shell specimens) were used in this study. The chitin content of the pupa shells on a dry basis was calculated as $\sim 26.4 \%$. The properties of the isolated chitin such as chemical bonding, thermal stability, crystallinity, hydrophobicity, and surface morphologies are given in the Supporting Information. All analysis results provided in the Supporting Information confirmed the purity of the chitin from BSF pupa shells.

Chitin Isolation. The chitin isolation from BSF pupa shells was carried out following the method reported by Kaya et al. ${ }^{11}$ The BSF pupa shell sample was washed with distilled water to remove any dirt particles that may remain on it before extracting the chitin and then dried at $50{ }^{\circ} \mathrm{C}$ for 3 days. The samples were subjected to very sensitive conditions in order not to disturb their original $3 \mathrm{D}$ structures. To remove the minerals from the samples, they were treated for $6 \mathrm{~h}$ in $1 \mathrm{M} \mathrm{HCl}$ solution at $60{ }^{\circ} \mathrm{C}$. The samples were then washed with distilled water until they reached neutral $\mathrm{pH}$ and dried in the oven at $50{ }^{\circ} \mathrm{C}$ for 3 days. After the demineralization process, the dried sample was treated with $1 \mathrm{M} \mathrm{NaOH}$ solution at $80{ }^{\circ} \mathrm{C}$ to remove the proteins from the structure. Following this procedure of deproteinization, the samples were washed with distilled water until neutral $\mathrm{pH}$ was achieved and oven-dried at $50{ }^{\circ} \mathrm{C}$ for 3 days. The purity and properties of the obtained chitin were confirmed by Fourier transform infrared (FT-IR) (Figure S1), thermogravimetric analysis (TGA) (Figure S2), contact angle (Figure S3), and scanning electron microscopy (SEM) analyses (Figure S4), which are provided in the supplementary document. The functional group and vibration modes of NCFs are shown in Table S1.

Production of Films. Natural Chitosan Film Production. Pure 3D chitin films isolated from BSF pupa shells were refluxed with $60 \%$ $\mathrm{NaOH}$ for $5 \mathrm{~h}$ at $130{ }^{\circ} \mathrm{C}$ for chitosan production. After this process, samples were washed with distilled water until they reached a neutral $\mathrm{pH}$. The 3D chitosan films were initially produced in the form of a pouch and then were cut with a scalpel. The films were dried flat by holding them between two microscope slides.

Semisynthetic Chitosan Film Production and Characterization. A method reported in our previous studies for SCF production was followed with some modification. ${ }^{22} 200 \mathrm{mg}$ of chitosan produced from BSF pupa shells were dissolved in a $20 \mathrm{~mL}$ of $1 \%$ acetic acid solution $(0.018 \mathrm{M}, \mathrm{pH}=3.25)$ with a magnetic stirrer for 2 days. 100 $\mu \mathrm{L}$ of glycerol was added to the resulting gel as a plasticizer. Besides, 5 $\mu \mathrm{L}$ of glutaraldehyde was added as a cross-linker to obtain a homogeneous film and mixed with the homogenizer until it became homogeneous. Then, the film-forming solution was poured into a Petri dish $(60 \times 15 \mathrm{~mm})$ and dried for 3 days at room temperature at $25 \pm 1{ }^{\circ} \mathrm{C}$. The thickness of the produced films was measured using a Coolant Proof Micrometer-293 Mitutoyo (USA). The raw pupa shells, isolated chitin, and obtained chitosan are shown in Figure S5. The isolated natural and synthetic films were characterized physicochemically using various analytical tools such as FT-IR, Xray diffraction (XRD), TGA, contact angle, and elemental analysis with the degree of deacetylation (DD). The dissolution of the NCF in $1 \%$ acetic acid is shown in Figure S6. Furthermore, soil and water degradation assays were conducted to investigate the degradation of isolates. A detailed methodology has been provided in the Supporting Information.

Bacterial Attachment Study. Twelve clinical Escherichia coli isolates presenting multidrug resistance and producing biofilm were chosen for the study. To prepare inoculum for each $E$. coli sample, strains were first incubated at $37{ }^{\circ} \mathrm{C}$ for $24 \mathrm{~h}$ on LB agar (Merck, Germany) plates. Morphologically similar colonies were collected from Petri dishes and transferred into sterile saline solution $(0.85 \%$ $\mathrm{NaCl}$ ). The turbidity of bacterial suspensions was adjusted against the $0.5 \mathrm{McFarland}$ standard.

Glucose concentration is known to affect biofilm formation significantly. To determine the optimum glucose concentration and E. coli strain to be used in the study, the biofilm production capacities of each strain were tested in a Tryptic soy broth (TSB) containing glucose ranging between 0.00 and $1.25 \% .^{23}$ All E. coli strains were incubated at $37{ }^{\circ} \mathrm{C}$ for 24 and $48 \mathrm{~h}$ with five replicates, and a wellcontaining TSB medium was used as a negative control using 96-well plates. After the incubation period, the plates were washed by sterile distilled water $\left(\mathrm{sdH}_{2} \mathrm{O}\right)$ twice and air-dried at room temperature $(25$ $\pm 1{ }^{\circ} \mathrm{C}$ ). At the end of the drying process, a $1 \%$ crystal violet solution was transferred into the wells to stain the biofilm layer formed and the plates were incubated for $30 \mathrm{~min}$ at room temperature. ${ }^{24}$ The plates were again washed by $\mathrm{sdH}_{2} \mathrm{O}$ twice and air-dried. An ethanolacetone (70:30) solution was transferred into each well and incubated at room temperature for $30 \mathrm{~min}$ to dissolve crystal violet that stained the biofilm layer. The content of each well was transferred into a new plate, the absorptions of each well were recorded at $595 \mathrm{~nm}$ by a plate reader (BioTek), and the results were used to determine the optimum 


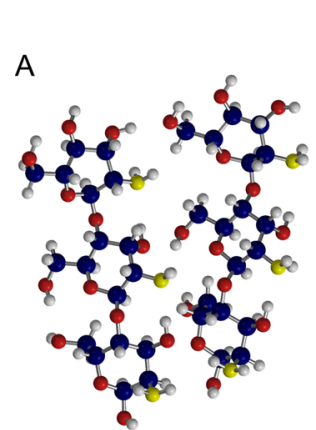

Natural Chitosan Film

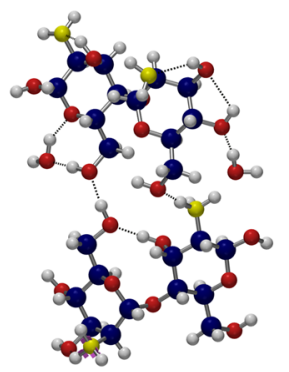

Semi-synthetic Chitosan Film
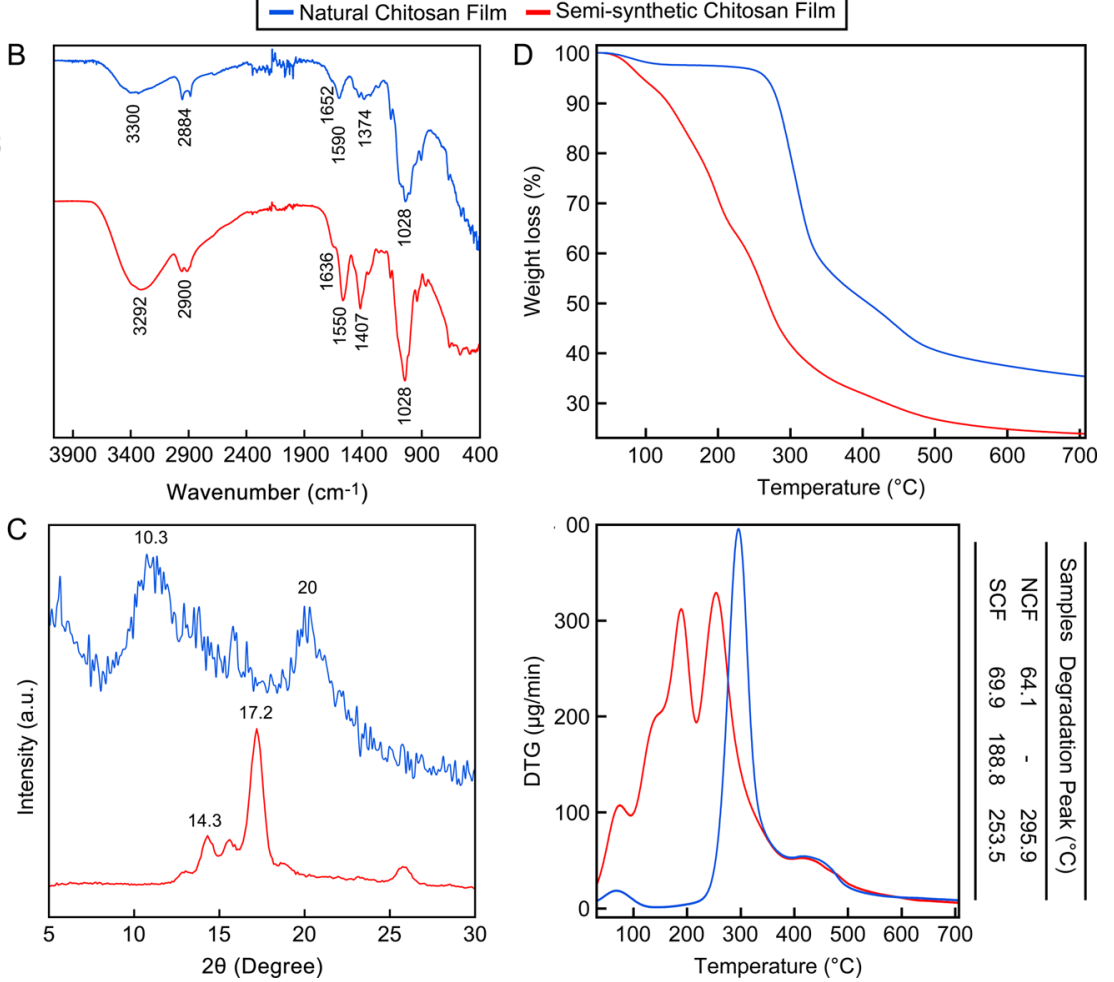

Figure 1. Characterization of NCFS and SCFs. The blue color represents the natural film, while the red color is the synthetic one. (A) Molecular structure of the natural and semisynthetic film, (B) FT-IR spectra, (C) XRD pattern, and (D) TG and derivative thermogravimetry (DTG) curves.

glucose concentration, incubation time, and E. coli strain to be used in the second part of the study. ${ }^{25}$

For testing biofilm forming E. coli, strains on sterilized synthetic and NCFs were placed into wells of 24-well plates, which contained TSB with optimum glucose concentration $(0.5 \%)$ and E. coli inoculum, and incubated at $37^{\circ} \mathrm{C}$ for $48 \mathrm{~h}$. The films were examined by SEM according to the procedure stated by Asahi et al. ${ }^{26}$

Deposition of Ag Nanofilms and Characterization of SERS Activity. Thermal evaporation (NanoVak) was used to deposit Ag on top of the NCFs and SCFs that were fixed on glass substrates. The deposition was performed via a $10 \mathrm{rpm}$ rotation at a rate of $0.4 \AA / \mathrm{s}$ with a total thickness of $\sim 50 \mathrm{~nm}$. The system was evacuated to a pressure of $2 \times 10^{-8}$ Torr following loading of the chitosan films to the chamber. An Ag pellet with a purity of $99.99 \%$ was used for the deposition.

The SERS activity of the NCFs and SCFs with Ag nanofilms was studied with a confocal Raman microscope (alpha M+, WITec, Germany) equipped with a $532 \mathrm{~nm}$ laser. $1 \mu \mathrm{L}$ of an $1 \mathrm{mM}$ aqueous solution of methylene blue (MB) was drop-casted on the natural and synthetic chitosans with the film of Ag. Raman measurements were then performed after the complete evaporation of water. The Raman spectra were measured using a $50 \times$ objective with a numerical aperture value of 0.85 . The integration time was set as $0.05 \mathrm{~s}$, and the power of the laser was $0.1 \mathrm{~mW}$. The SERS activity of the surfaces was mapped at a spatial resolution of $0.5 \mu \mathrm{m}$ in an area of $80 \times 80 \mu \mathrm{m}^{2}$ using the characteristic peak of MB located at a Raman shift of 1625 $\mathrm{cm}^{-1}$. To determine the limit of detection of the NCFs with an $\mathrm{Ag}$ nanofilm, the concentration of MB varied between $100 \mathrm{nM}$ and $1 \mathrm{pM}$. In the limit of the detection study, the SERS spectra were collected from at least 10 different spots with an integration time of $0.5 \mathrm{~s}$.

Molecular Modeling Studies. In the present study, three computational methods were used to determine the stability of chitosan in different conditions. The molecular long chain of chitosan is the repetition of $\mathrm{CD}$. To reduce the computational cost, the $\mathrm{CD}$ was chosen as a model and the following steps were performed to determine the stability of the macromolecule: (i) to determine the optimum configurations of $\mathrm{CD}$ which corresponds to the NCF and
CDA, ACD, and PACD which correspond to semisynthetic films, conformational analysis was carried out through a molecular mechanics MMFF model using Spartan'18 Software; (ii) to investigate the molecular interactions of $\mathrm{CD}$ and acetic acid, the lowest energy conformers of $\mathrm{CD}$ with $\mathrm{CDA}$ were chosen and reoptimized using Hartree-Fock 6-31G*; (iii) to determine the stability of the ACD in acetic acid solvent, the polarizable continuum solvation model was performed using Hartree-Fock 6-31G* for the lowest energy conformer of ACD; and (iv) to compare the stability of PACD dissolved in acetic acid solution with CD, CDA, and ACD, the experimental conditions were simulated and the polarizable continuum solvation model was performed to PACD at the Hartree-Fock 6-31G* level. In this context, many questions arising from the conformational analysis and polarizable continuum solvation models of $\mathrm{CD}, \mathrm{ACD}, \mathrm{CDA}$, and PACD have been addressed to compare their stabilities and to provide better insights into the interaction mechanism of chitosan and acetic acid. More detailed information about molecular modeling is presented in the Supporting Information.

\section{RESULTS AND DISCUSSION}

Characterization of Natural and Semisynthetic Chitosan Films. The molecular structures of the natural and semisynthetic films using simulation techniques are provided in Figure 1A. FT-IR results of natural and semisynthetic films are given in Figure 1B. It is visible that two characteristic peaks for chitosan (amide I: $1652 \mathrm{~cm}^{-1}$ and amide II: $1590 \mathrm{~cm}^{-1}$ ) indicated the deacetylation of the chitin by successfully keeping the original 3D film shape. After dissolving this chitosan film in acetic acid, the semisynthetic film was produced by adding glycerol as a plasticizer and glutaraldehyde for cross-linking. It was observed that the FTIR spectrum changed almost completely as a result of the conversion of NCFs to semisynthetic films. The $3300 \mathrm{~cm}^{-1}$ band observed in the natural film was recorded as a violent and 
A

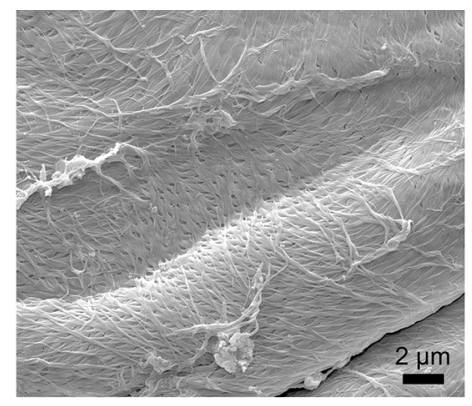

B

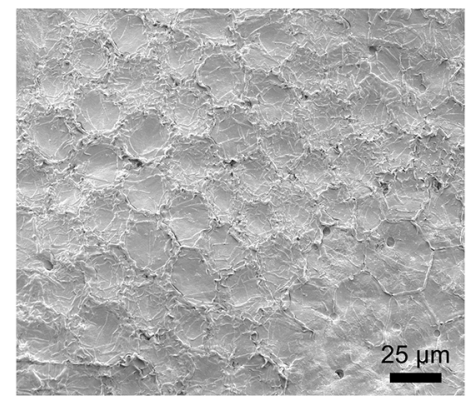

C

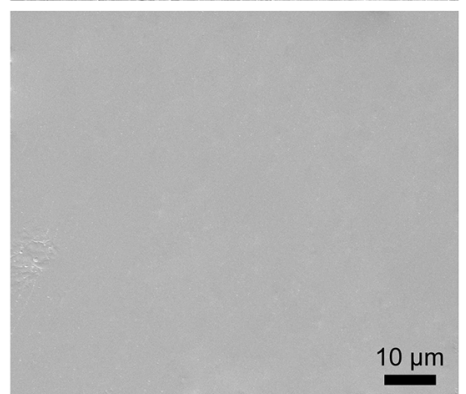

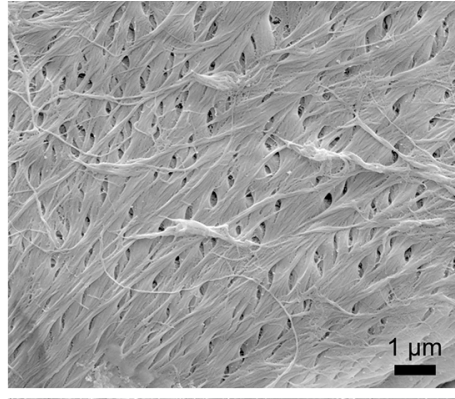
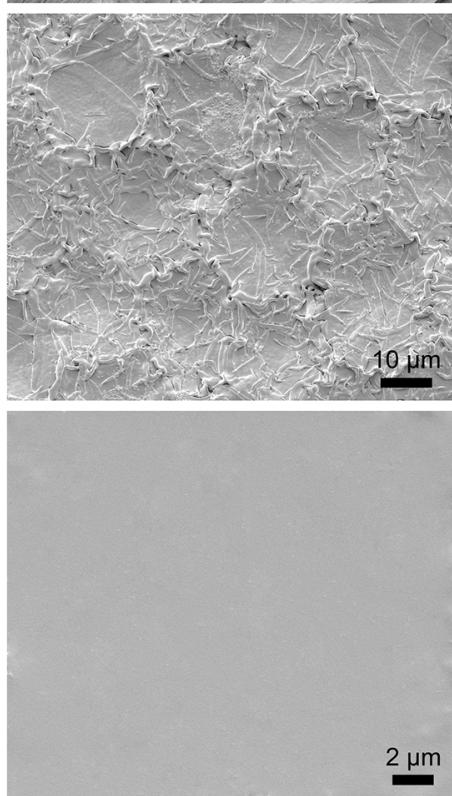

D

Natural Chitosan Film Inner

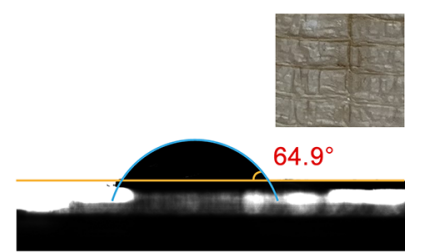

Natural Chitosan Film Outer

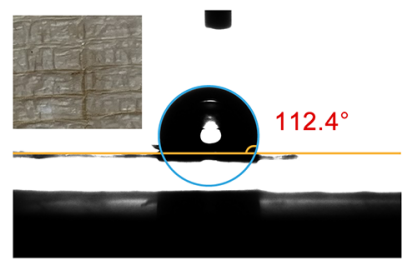

Semi-synthetic Chitosan Film
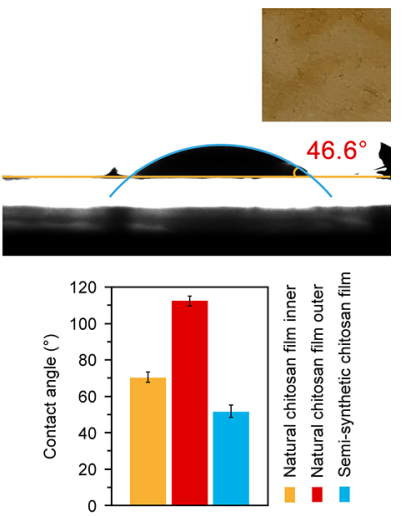

Figure 2. SEM images: (A) inner surface of the NCF, (B) outer surface of the NCF, (C) surface of the SCF, and (D) water contact angle measurements.

wideband at $3292 \mathrm{~cm}^{-1}$ in the synthetic film due to the presence of glycerol and water by increasing the $\mathrm{O}-\mathrm{H}$ concentration in the synthetic film. The $\mathrm{N}-\mathrm{H}$ bending peak at $1590 \mathrm{~cm}^{-1}$ is a well-known characteristic peak for chitosan which is extensively reported in the literature. ${ }^{27}$ Here, in this study for natural film, this characteristic peak was recorded as $1590 \mathrm{~cm}^{-1}$; however, in the case of semisynthetic film, this peak demonstrated a huge shift from 1590 to $1550 \mathrm{~cm}^{-1}$ with an increased intensity due to the formation of hydrogen bonds between $\mathrm{N}-\mathrm{H}$ groups and glycerol, glutaraldehyde, or water. A detailed comparison between the spectra of natural and semisynthetic films, including the related literature results, has been provided in Table S2. The $\mathrm{C}-\mathrm{N}$ stretching vibration observed at $1374 \mathrm{~cm}^{-1}$ in the natural film shifted to the lower wavenumber $\left(1335 \mathrm{~cm}^{-1}\right)$ due to the effect of the molecular interactions between compounds in the semisynthetic films. The FT-IR data clearly explained the changes that occurred in the polymeric structure of chitosan followed by its dissolution in acetic acid.

$\mathrm{XRD}$ results of natural and semisynthetic films are given in Figure 1C. In the XRD analysis of NCFs, two sharp peaks were observed at 10.3 and $20^{\circ}$, which are characteristic for chitosan. ${ }^{28}$ In the semisynthetic film, these peaks are shifted to 14.3 and $17.2^{\circ}$. This result showed that the crystalline structure of the NCF was changed after making it a semisynthetic film. The alternation in the crystalline structure in semisynthetic films can be attributed to the incorporation of a plasticizer. According to Bourtoom, ${ }^{29}$ the incorporation of a plasticizer into the chitosan film may cause the ordering of polymeric chains as per their degree of polymerization, enhancing the chain interaction which leads to an increase in the crystalline structure. As is known that during XRD analysis, every phase results in a different diffraction pattern. Each phase is specific to the chemistry and atomic arrangement of chemically identical materials. In the current study, the same situation is valid for NCFs and semiSCFs. As it is also evident from the molecular modeling of both the films, chitosan undergoes a series of chemical rearrangements after dissolution in acetic acid. Besides, the incorporation of the plasticizer contributes to the chemical rearrangement during the process. As a result, this chemical rearrangement reflected in the shift of $\mathrm{XRD}$ patterns, that is, peak at 11 shifted to 14 and peak at 20 shifted to 17.5. Considering the related literature reports, the shift in the first peak 11 from 14 can be ascribed to the hydration of chitosan molecules. ${ }^{30}$ This hydration has occurred during the dissolution of chitosan in $1 \%$ acetic acid solution (99\% water). ${ }^{31}$ The shift in the second peak is due to the change in the amorphous condition of chitosan due to the addition of acetic acid and glycerol.

Thermal properties of natural and semisynthetic films are given in Figure 1D. In NCFs, the mass losses occurred in two steps, while in semisynthetic films, it occurred in three steps. In both films, the first mass loss results from the evaporation of water in the structure. The initial mass loss was recorded as $2.81 \%$ for natural film and $6.85 \%$ for the semisynthetic film. The secondary mass loss was observed as $61.98 \%$ for the 

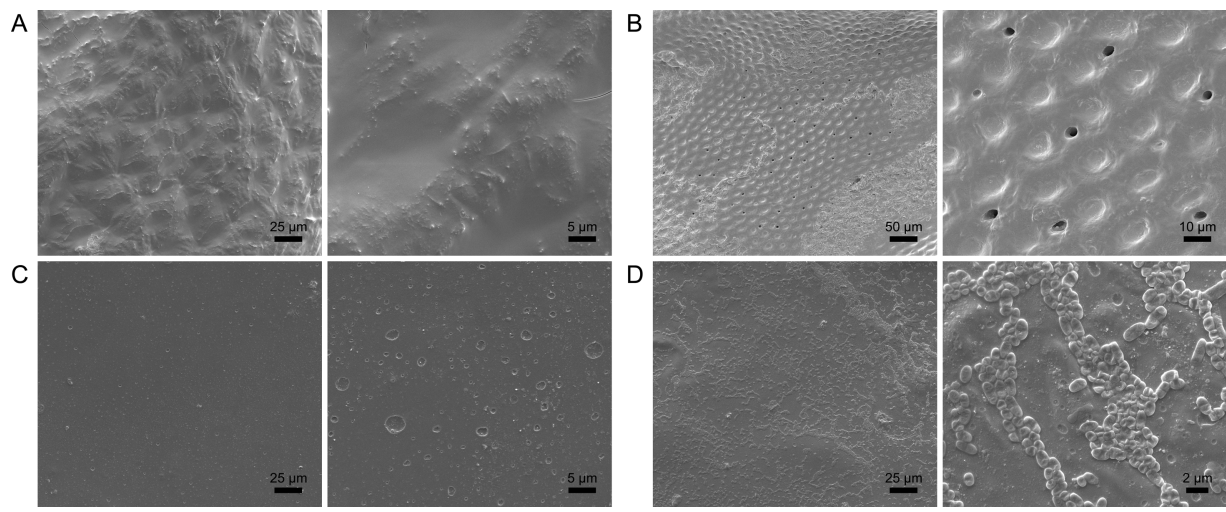

Figure 3. SEM images of (A) surface of NCFs in the culture medium, (B) surface of NCFs in culture medium + E. coli, (C) surface of SCF in the culture medium, and (D) surface of SCF in culture medium + E. coli.

natural film. This loss corresponds to the deterioration of saccharide structures, and the maximum deterioration was measured at $295.9{ }^{\circ} \mathrm{C}$. The second mass loss of the semisynthetic film is due to the deterioration of glycerol, and maximum deterioration $(29.52 \%)$ was measured at $188.8{ }^{\circ} \mathrm{C}$. These results were also in line with the previous findings by different literature reports. ${ }^{32,33}$ The third mass loss of the semisynthetic film was $40.0 \%$. This mass loss can be attributed to the breakdown of the polymeric structure, and the maximum degradation was measured at $253.5^{\circ} \mathrm{C}$. Considering the thermal analysis results, the thermal stability of the NCF was observed to be higher than the semisynthetic film. The alleviated thermal stability of the semisynthetic film can be attributed to the rearrangement of polymeric chains during acid hydrolysis and the possible protonation of amino groups of chitosan molecules. The protonation of chitosan amino groups in the film state enhances the affinity of the chitosan molecule for water. This increased water affinity results in the formation of interactions between water molecules, decreasing the overall stability of the structure and enhancing the chain mobility of chitosan films. ${ }^{34}$

According to the elemental analysis results, $\% \mathrm{~N}, \mathrm{C}$, and $\mathrm{H}$ values of the NCF were recorded as $7.3,42.8$, and $6.8 \%$, respectively. Theoretically, the nitrogen value of chitin is known as $6.89 \% .{ }^{35}$ Here, in this study, a \% $\mathrm{N}$ value which is higher than 6.89 indicates that the chitin was successfully converted to chitosan. Furthermore, the DD value of the NCF was calculated as $58 \%$. This value indicates that there are still $\mathrm{N}$-acetyl groups in the structure of chitosan. Although different conditions were tried before, results revealed that the $N$-acetyl groups could not be completely removed from the structure. ${ }^{36}$ In the same regard, here in the current study, we predict that the complete removal of these groups from NCFs is nearly impossible. For chitosan, the degree of acetylation (the ratio of $\mathrm{N}$-acetyl-D-glucosamine units to the total number of units) is considered to be less than $50 \%{ }^{37}$ In the present study, the acetylation degree of the naturally obtained chitosan film was calculated as $42 \%$. On the other hand, $\% \mathrm{~N}, \mathrm{C}, \mathrm{H}$, and $\mathrm{O}$ values of the semisynthetic film were recorded as 4.4, 39.6, 6.7, and 49.3, respectively. Elemental analysis results showed that $\mathrm{N}$ and $\mathrm{C}$ values changed with the conversion of natural chitosan to the semisynthetic film. When compared with the oxygen values of natural and semisynthetic films, the $O$ value of the synthetic film was recorded to be higher than the natural film. For the preparation of the semisynthetic film, glycerol, acetic acid $(1 \% \mathrm{v} / \mathrm{v})$ solution, and glutaraldehyde were blended with chitosan and some changes were observed in the molecular structure due to the molecular interactions. These molecular interactions can be observed from the $\mathrm{O}-\mathrm{H}$ band widening at the FT-IR spectra in Figure 1B. The widening of the $\mathrm{O}-\mathrm{H}$ band at the IR spectrum usually occurs in the presence of water and the formation of $\mathrm{H}$ bonds. The present water in the acetic acid solution has interacted with the polymer chains of the chitosan. Thus, the water bonded to $\mathrm{OH}$ and $\mathrm{NH}_{2}$ groups of polymer chains of chitosan with hydrogen bonds during gel formation. While the semisynthetic film is being prepared, bonded water did not leave the gel matrix and the percentage of oxygen increased. An increase in the oxygen percentage decreased the $\mathrm{C}$ and $\mathrm{N}$ percentage of the semisynthetic film.

Surface morphologies of natural and semisynthetic films were unveiled with SEM (Figure $2 \mathrm{~A}-\mathrm{C}$ ). It was observed that the inner surface of the NCF consists of nanofibers and the presence of pores in some parts (Figure 2A). The unique hexagonal structures on the outer surface of the natural chitin film (Figure S4) manifested itself in the natural chitosan form with slight deformation (Figure 2B). Some previous studies reported that the surface morphology of powder chitosan (without dissolving in acetic acid) obtained from silkworm chrysalides, shrimp, and crab shells presented similar structural morphologies with fibrils and pores. ${ }^{38-40}$ However, in the present study, no fibers, pores, or any specific structure were observed on both sides of the SCF (Figure 2C). These results were also supported by some previous literature reports where semisynthetic chitosan-based films demonstrated fibreless, smooth, and compact surface morphologies. ${ }^{41-44}$ Contact angle measurements (Figure 2D) demonstrated that the inner surface of the film has a hydrophilic character with a water contact angle of $64.9^{\circ}$, but the outer surface is hydrophobic with an angle of $112.4^{\circ}$. The surface of the synthetic film was found to be hydrophilic with an angle of $46.6^{\circ}$ (Figure 2D). As shown from the results, the NCF has two different sides, inner and outer, and their morphologies differ from each other. These differences in the surface morphology and contact angle may be an important feature for the future technological applications of the films.

Degradability in Water and Soil. The solubility of NCFs and SCFs was investigated in water after $48 \mathrm{~h}$, and no weight loss was recorded for the natural film; however, $34 \%$ of mass loss was observed for the semisynthetic film (Figure S7). The NCF retained its original shape after keeping it in the soil for 60 days without any mass loss. On the other hand, $86.8 \%$ of the semisynthetic film is degraded in the soil in 60 days (Figure 
A

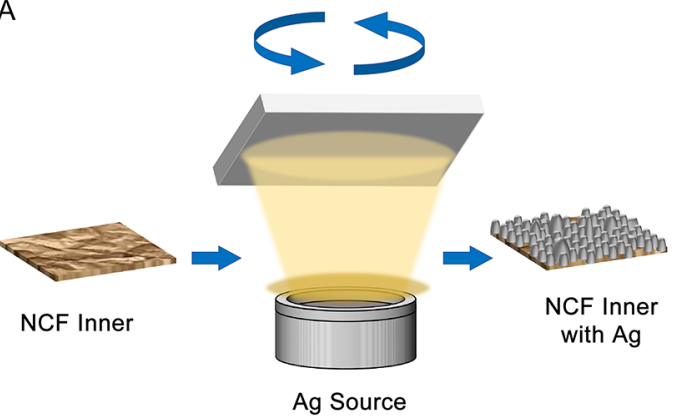

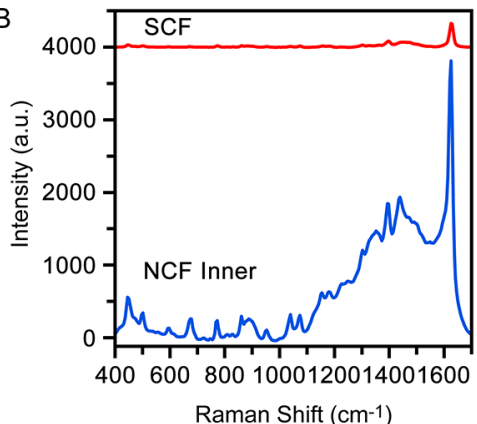
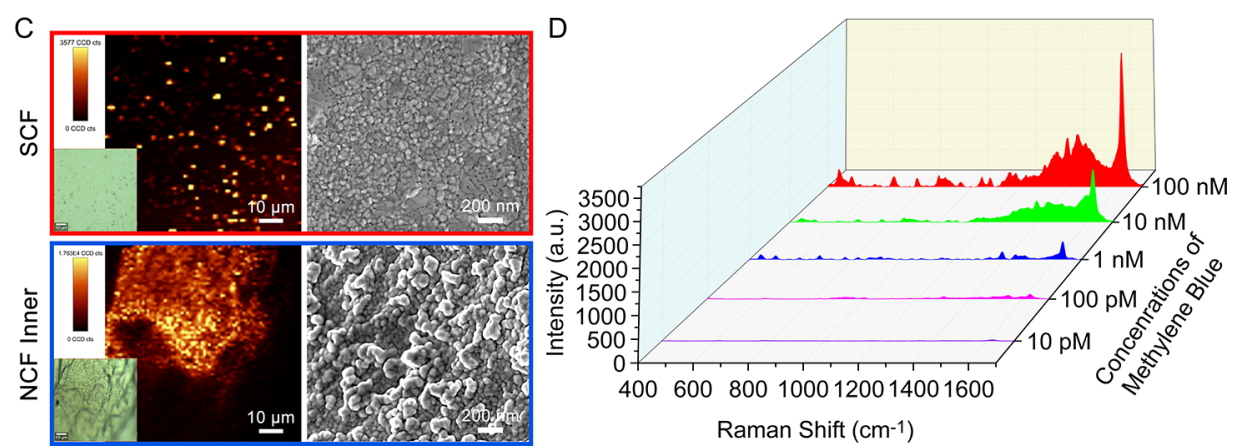

Figure 4. SERS activity of NCFs and SCFs. (A) Schematic description of the physical vapor deposition of Ag films on NCFs and SCFs. (B) Raman spectra of MB deposited from $1 \mathrm{mM}$ aqueous solution on the Ag-deposited NCFs and SCFs. (C) Raman mapping and SEM images of the Ag deposited SCFs and NCFs, respectively. The insets present optical microscopy images of the samples. (D) Concentration study. SERS spectra of $\mathrm{MB}$ for concentrations that range from $100 \mathrm{nM}$ to $10 \mathrm{pM}$.

S8). The degradability properties of the SCF were observed to be increased, as compared to the NCF. This can be ascribed to two main factors, acid hydrolysis of semisynthetic film and incorporation of a hygroscopic plasticizing agent (glycerol). The SCF becomes more soluble because, after dissolution in acetic acids, the salts of the acid remain inside the Dglucosamine units. The presence of acid salts provides more binding sites for water molecules, and as a result, enhancing the overall solubility of SCFs. ${ }^{45}$ The increase in soil degradability can be attributed to the incorporation of glycerol as a plasticizing agent. It is known that glycerol is a strong swelling reagent, swelling the SCF in soil conditions. This swelling of the semisynthetic film results in a loose polymeric chain network, making it easy for enzymes (present under soil conditions) to invade into the film structure. ${ }^{46}$ The water and soil degradability results of the current study were recorded, in line with previous literature reports. ${ }^{47,48}$

Bacterial Attachment on the Surface of the Films. It is a well-known issue that biofilms have great importance in the surface attachment of bacteria. Bacterial cells, which will transform from a free-swimming mode of growth to growth by attaching to a surface, form a monolayer on a surface and then start to form an extracellular matrix, to which they attach themselves irreversibly. ${ }^{49}$ Thus, in the first part of the bacterial attachment test, an E. coli strain having the capability of producing biofilms was chosen. $48 \mathrm{~h}$ of incubation time was previously proposed by several studies to be a good time point to observe bacteria attached to any surface with sufficient quantities but without forming a significant biofilm which may affect the results. ${ }^{50}$ The SEM photographs given in Figure 3 show whether E. coli can successfully attach to the surface of natural and semisynthetic films.

Figure 3A presents the surface of the NCF after incubating in a glucose-containing culture medium, where $3 \mathrm{~B}$ presents the surface of the same film after incubating glucose-containing culture medium with E. coli strain. The results clearly show that E. coli did not attach to the surface of the NCF. Figure 3C shows the surface of SCF incubated in only a culture medium, and Figure 3D shows the surface of the same film incubated in the medium containing E. coli strain. As a result, it can be proposed that the biofilm-producing E. coli strain did not attach to the NCF surface but did so to the SCF.

Surface-Enhanced Raman Scattering Activity. The striking contrast in the structure of the NCFs and SCFs can lead to a significant difference in their properties. One particular area of interest is SERS, which is a label-free spectroscopic technique with demonstrated utility in sensing drug molecules, biomarkers, pollutants, and so forth. ${ }^{51}$ The most exploited mechanism of SERS involves the electromagnetic enhancement of the weak Raman scattering signals. Rough metal nanostructures have received enormous interest as SERS substrates, with their ability to contain plasmonic hotspots, where electromagnetic fields are concentrated. ${ }^{52}$ Therefore, we hypothesized that the deposition of a metallic film on top of the NCFs and SCFs should lead to a significant difference in their SERS activity. For this purpose, physical vapor deposition was used to deposit a nanoscopic film of $\mathrm{Ag}$ NCFs and SCFs (Figure 4A). The SERS activity was then investigated using a commonly used probe molecule, MB.

Figure $4 \mathrm{~B}$ presents the average SERS spectra of $\mathrm{MB}$ on the backside of the synthetic and inner side of the NCFs. The intensity of Raman scattering signals of $\mathrm{MB}$ was significantly higher for the NCFs in comparison to synthetic ones. The intensity at a position of $1625 \mathrm{~cm}^{-1}$, which is related to the carbon-carbon ring stretching, was 12 -fold higher on the $\mathrm{Ag}$ deposited natural film. This result suggests that the deposition of a metallic film on the natural structure of chitosan film favors the formation of plasmonic hot-spots. Figure 4C clearly 
A
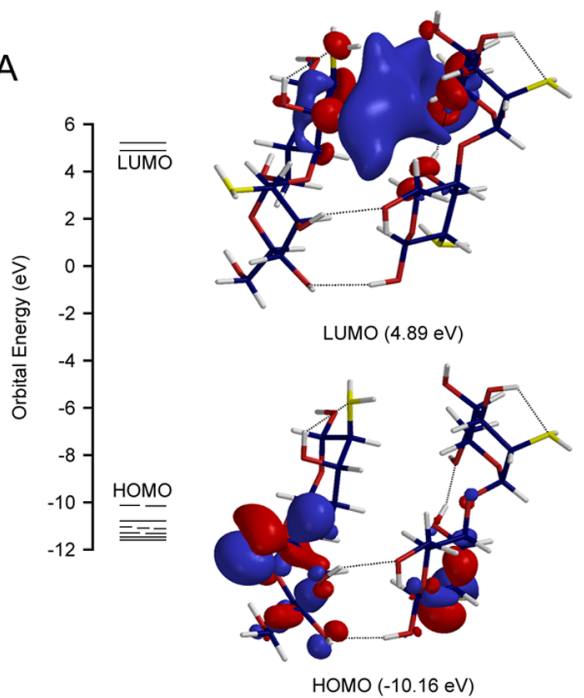

C
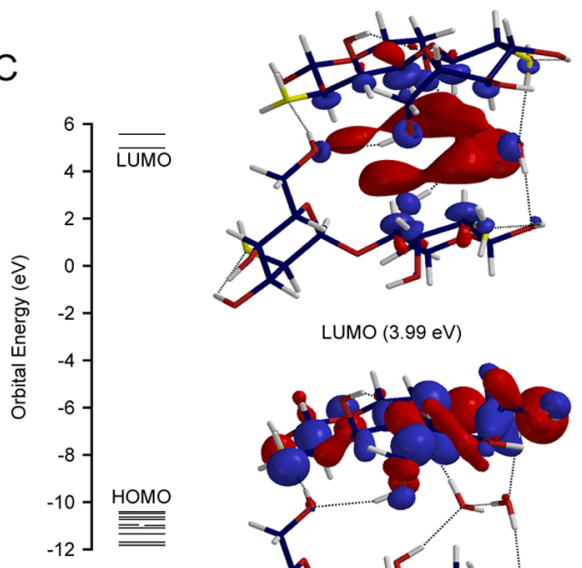

LUMO $(3.99 \mathrm{eV})$

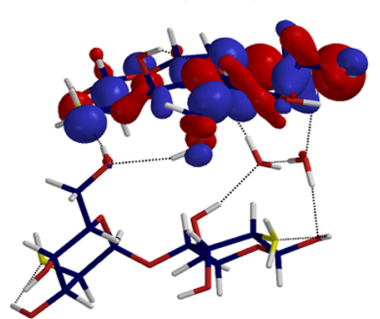

HOMO $(-10.44 \mathrm{eV})$

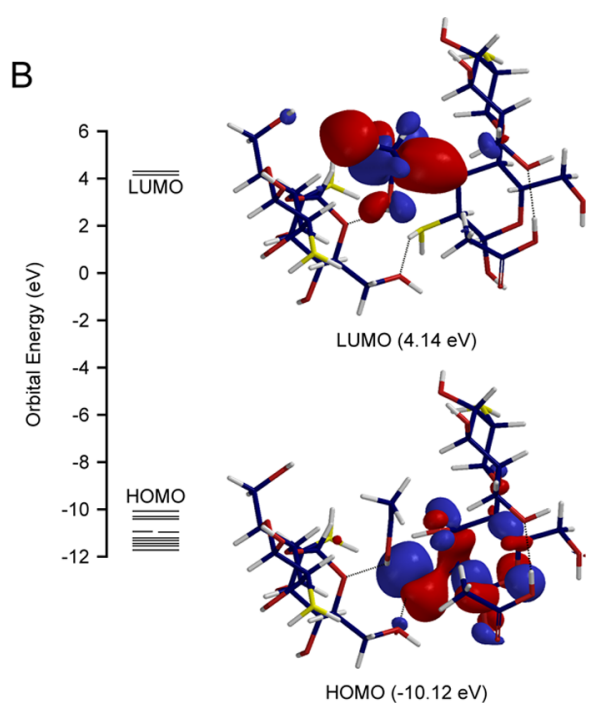

D

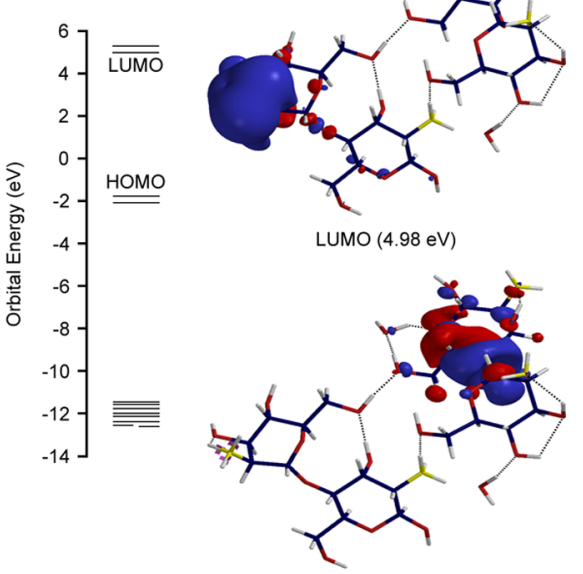

HOMO $(-1.79 \mathrm{eV})$

Figure 5. Molecular orbital surfaces for the HOMO and LUMO of (A) CD, (B) CDA, (C) ACD, and (D) PACD computed at the Hartree-Fock 6-31G* level. Energy levels of HOMO and LUMO are given in parentheses.

shows the strong contrast in the morphology of the natural and synthetic films following the deposition of a layer of Ag. The natural film exhibited a texture with distinct nanostructures. The loss of this unique structure in the SCFs manifests itself as reduced SERS activity. Raman mapping images together with SEM images present the correlation between surface morphology and plasmonic properties. In the case of SCFs, the surface acts like a planar substrate with low SERS activity. ${ }^{53}$ It is known from previous studies that sharp edges, as well as close placement of metallic structures, result in improved SERS activity. ${ }^{54,55}$

Two figures of merit are commonly used to evaluate the SERS performance of platforms. One is the limit of detection, which shows the lowest concentration that can be detected in the Raman spectroscopy over the substrate of interest. To find the limit of detection, we deposited different concentrations of $\mathrm{MB}$. Figure $4 \mathrm{C}$ shows that the $\mathrm{MB}$ at concentrations as low as $10 \mathrm{pM}$ can be detected on the NCFs with a nanoscopic Ag film. The analytical enhancement factor (AEF) for the probe molecule of $\mathrm{MB}$ was calculated by the following equation

$$
\mathrm{AEF}=\frac{I_{\mathrm{SERS}}}{I_{\mathrm{RM}}} \times \frac{C_{\mathrm{RM}}}{C_{\mathrm{SERS}}}
$$

The reporter molecule with a volume of $2 \mu \mathrm{L}$ and a concentration of $1 \mathrm{mM}\left(C_{\mathrm{RM}}\right)$ dropped cast on a glass slide, which served as the reference substrate. The Raman intensity of the reporter molecule was measured on the Ag deposited natural chitosan $\left(I_{\text {SERS }}\right)$ and bare glass substrate $\left(I_{\mathrm{RM}}\right)$. The AEF was calculated as $8.14 \times 10^{6}$ with $I_{\text {SERS }}\left(25\right.$ counts), $I_{\mathrm{RM}}$ (307 counts), $C_{\text {SERS }}(10 \mathrm{pM})$, and $C_{\mathrm{RM}}(1 \mathrm{mM})$. This result is quite reasonable considering previous studies. This AEF is slightly higher than those obtained on a natural dragonfly wing with a layer of sputter-coated $\mathrm{Ag}$ film. ${ }^{56}$ The AEF value is also comparable with substrates obtained with wet-chemical methods. ${ }^{57,58}$

In the present study, conformational analysis using the molecular mechanics MMFF model was carried out for the determination of the conformational behavior of chitosan and stereoelectronic effects on the stability of chitosan. The heavily populated conformers of $\mathrm{CD}, \mathrm{CDA}, \mathrm{ACD}$, and $\mathrm{PACD}$ were determined as $5,13,17$, and 11 , respectively. The lowest 

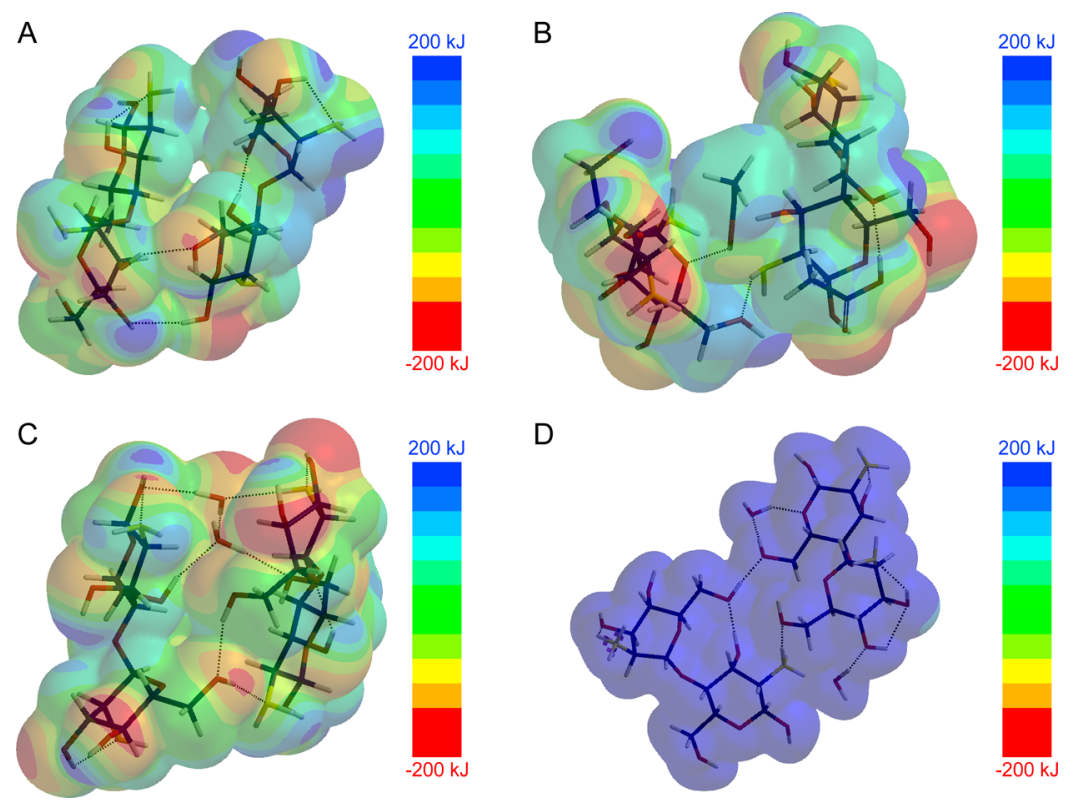

D

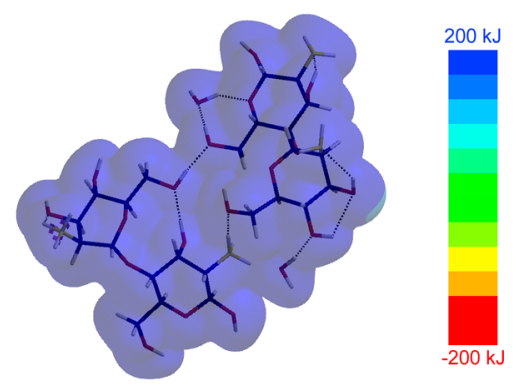

Figure 6. MEP map of (A) CD, (B) CDA, (C) ACD, and (D) PACD computed at the Hartree-Fock 6-31G* level. In the MEPs of the CD, CDA, and $\mathrm{ACD}$, the negative regions (red) existed at oxygen atoms and the positive regions (blue) existed at nitrogen atoms.

energy conformers given in Figure S9 of the called structures were reoptimized using a Hartree-Fock 6-31G*. The electrostatic and topological properties of the lowest energy conformers of CD, CDA, ACD, and PACD were computed and given in the Supporting Information. Besides, inter- and intramolecular $\mathrm{H}$ bonds were investigated for all structures.

In this section, the stability of $\mathrm{CD}, \mathrm{CDA}, \mathrm{ACD}$, and PACD was investigated by different parameters including Mulliken charges, bond lengths, bond angles, highest occupied molecular orbital (HOMO), lowest unoccupied molecular orbital (LUMO), Gibbs energy $\left(G^{\circ}\right)$, enthalpy $\left(H^{\circ}\right)$, entropy $\left(S^{\circ}\right)$, and molecular electrostatic potential (MEP). HOMO, LUMO, $G^{\circ}, H^{\circ}, S^{\circ}$, and MEP were calculated and are given in Table S3. According to Table S3, the lowest and the highest $G^{\circ}$ values were determined for $C D$ and PACD, respectively. Since the lower $G^{\circ}$ value implies the more stable state for chemical molecules, the $\mathrm{CD}$ is the most stable structure among all investigated structures.

It was determined that two intermolecular $\mathrm{H}$ bonds were formed between $\mathrm{CD}$ in the gas phase, whereas only one intermolecular $\mathrm{H}$ bond was found in the CDA. The acetic acid molecule caused the breakage of the intermolecular $\mathrm{H}$ bonds between $C D$. The stability of the $C D$ was decreased by the bonding of the acetic acid to chitosan via a new $\mathrm{H}$ bond. In the solvent phase, two water molecules were used to simulate the experimental conditions. In this regard, the water molecules were bonded to $\mathrm{CD}$ with four and three $\mathrm{H}$ bonds in $\mathrm{ACD}$ and $\mathrm{PACD}$, respectively. Besides, intermolecular $\mathrm{H}$ bonds were determined between the $\mathrm{O}-\mathrm{H}$ and $\mathrm{N}-\mathrm{H}$ atoms in $\mathrm{CDs}$ of ACD and PACD. Additionally, the intramolecular $\mathrm{H}$ bonds were observed in all of the optimized structures.

When comparing the bond lengths (Table S4) of hydrogen bond acceptor (HBA) and hydrogen bond donor (HBD) atoms in all of the structures, it was determined that the formation of the $\mathrm{H}$ bonds caused bond lengths to change. In particular, the bond lengths between $\mathrm{O}-\mathrm{H}$ atoms in the PACD are longer than the $\mathrm{O}-\mathrm{H}$ atoms in the other structures. The bond angles and dihedral angles are given in Table S5 and varied according to the different structures. Dihedral angles of glycosidic linkages are one of the most important parameters to determine the conformational dynamics of chitosan. In the literature, it has been found that the torsional angles of chitin and chitosan are influenced by the ionic strength of the solvent. The dihedral angles of glycosidic linkages were of very different CDs in both gas and solvent phases. The differences between the torsion angles can be explained by high conformational exchange and flexibility.

The Mulliken charges and bond orders of HBA and HBD atoms computed for $\mathrm{CD}, \mathrm{CDA}, \mathrm{ACD}$, and $\mathrm{PACD}$ are given in Tables S6. The atom labels of CD, CDA, ACD, and PACD are given in Figure S9. It was seen that the Mulliken charges and bond order values were different for each of the structures. The formation of the hydrogen bonds and acetic acid-CD interaction was also evidenced by the bond order values of HBA and HBD atoms with a blue color in the given Table S6.

Frontier molecular orbitals referred to as the HOMO as an electron donor and the LUMO as an electron acceptor are responsible for the chemical reactions and electric and optical properties of molecules. The energy gap between HOMO and LUMO has been used for the determination of chemical stability, bioactivity, and chemical reactions. ${ }^{59,60}$ In the present study, the frontier molecular orbitals of CD, CDA, ACD, and PACD and their energy values are given in Figure 5. The energy gap between the HOMO and LUMO for CD, CDA, ACD, and PACD was calculated as $-15.05,-14.26,-14.43$, and -6.77 , respectively. The high difference between HOMO and LUMO expresses high chemical stability; the CD has the highest chemical stability and PACD has the lowest.

The MEP, which is generated from the electrons and nuclei of the molecules, is a very important parameter in understanding the hydrogen bonding interactions. In the present study, the MEPs of $\mathrm{CD}, \mathrm{CDA}, \mathrm{ACD}$, and PACD are given in Figure 6. As seen in Figure 6, the MEPs of the CD, CDA, and ACD showed that negative regions existed at the oxygen atoms, and the positive regions existed at the nitrogen atoms, while the positive regions of PACD were completely dispersed 
on the molecule. As the positive and negative regions in any molecule demonstrate the reactive sites of nucleophilic and electrophilic attacks for molecules, it can be remarked that the PACD was the most active among the chitosan structures. In other words, the reactivity of the PACD structure can be commented upon as having the lowest stability when comparing to $\mathrm{CD}, \mathrm{CDA}$, and $\mathrm{ACD}$ structures.

\section{CONCLUSIONS}

This study has demonstrated that favorable characteristics of the NCF are lost during dissolution in acetic acid including (i) a lack of the unique fibrous structure, (ii) decrease in the thermal stability, (iii) shift from hydrophobicity toward hydrophilicity, (iv) alteration in the crystal structure, (v) quick soil degradation, (vi) loss of antimicrobial surface features, and most importantly, (vii) the transformation of its polymeric structure into a different material. The molecular modeling studies demonstrated that the semisynthetic film was observed to be more reactive than the NCF in terms of electrostatic and topological parameters. The molecular modeling studies were recorded, highly in line with the other experimental results in the literature. Moreover, the $\mathrm{H}$ bonds formed between HBA and HBD atoms in the chitosan film were observed to affect the stability of natural and semisynthetic films. The abovementioned features of chitosan can be termed as determinative characteristics for the application of chitosan films in different industries such as pharmaceutics, cosmetics, agriculture, and biomedicine. To avoid such a major loss in the chemical and biological properties due to dissolution in acetic acid, and to exploit the real potential of chitosan films in the mentioned industries, the focus needs to be diverted toward the production of NCFs. From all these results, it can be concluded that the dissolved chitosan in acetic acid does not represent the properties of chitosan.

\section{ASSOCIATED CONTENT}

\section{sI Supporting Information}

The Supporting Information is available free of charge at https://pubs.acs.org/doi/10.1021/acssuschemeng.0c06373.

Detailed methodology for FT-IR, TGA, XRD, contact angle, water degradability, soil degradability, molecular modeling analysis, and chitin physicochemical characterization (PDF)

\section{AUTHOR INFORMATION}

\section{Corresponding Author}

Murat Kaya - Department of Biotechnology and Molecular Biology, Faculty of Science and Letters, Aksaray University, 68100 Aksaray, Turkey; ○ orcid.org/0000-0001-69542703; Phone: +90-382-288-2116;

Email: muratkaya3806@yahoo.com

\section{Authors}

Ismail Bilican - Department of Electronics and Automation, Technical Vocational School, Aksaray University, 68100 Aksaray, Turkey; UNAM\National Nanotechnology Research Center, Institute of Materials Science and Nanotechnology, Bilkent University, 06800 Ankara, Turkey; (1) orcid.org/0000-0002-4415-6803

Sami Pekdemir - ERNAM\Erciyes University Nanotechnology Application and Research Center, 38039
Kayseri, Turkey

M. Serdar Onses - ERNAM冈Erciyes University Nanotechnology Application and Research Center, 38039 Kayseri, Turkey; Department of Materials Science and Engineering, Erciyes University, Kayseri 38039, Turkey

Lalehan Akyuz - Department of Chemistry Technology, Technical Vocational School, Aksaray University, 68100 Aksaray, Turkey

Ergin Murat Altuner - Department of Biology, Faculty of Science and Arts, Kastamonu University, 37150 Kastamonu, Turkey

Behlul Koc-Bilican - Department of Biotechnology and Molecular Biology, Faculty of Science and Letters, Aksaray University, 68100 Aksaray, Turkey

Lian-Sheng Zang - Jilin Engineering Research Center of Resource Insects Industrialization, Jilin Agricultural University, Changchun, China

Muhammad Mujtaba - Institute of Biotechnology, Ankara University, 06110 Ankara, Turkey; 이이.org/0000-00018392-9226

Povilas Mulerčikas - Vytautas Magnus University, 44248 Kaunas, Lithuania

Complete contact information is available at:

https://pubs.acs.org/10.1021/acssuschemeng.0c06373

\section{Notes}

The authors declare no competing financial interest.

\section{ACKNOWLEDGMENTS}

The authors thank ASUBTAM (Aksaray University) for providing laboratory facilities and access to equipment.

\section{REFERENCES}

(1) Wang, L.; Dong, Y.; Men, H.; Tong, J.; Zhou, J. Preparation and characterization of active films based on chitosan incorporated tea polyphenols. Food Hydrocolloids 2013, 32, 35-41.

(2) Elsabee, M. Z.; Abdou, E. S. Chitosan based edible films and coatings: A review. Mater. Sci. Eng. C 2013, 33, 1819-1841.

(3) Xu, Y. X.; Kim, K. M.; Hanna, M. A.; Nag, D. Chitosan-starch composite film: preparation and characterization. Ind. Crop. Prod. 2005, 21, 185-192.

(4) Bonilla, J.; Sobral, P. J. A. Investigation of the physicochemical, antimicrobial and antioxidant properties of gelatin-chitosan edible film mixed with plant ethanolic extracts. Food Biosci. 2016, 16, 17-25.

(5) Huang, J.; Qin, J.; Zhang, P.; Chen, X.; You, X.; Zhang, F.; Zuo, B.; Yao, M. Facile preparation of a strong chitosan-silk biocomposite film. Carbohydr. Polym. 2020, 229, 115515.

(6) Peng, Y.; Li, Y. Combined effects of two kinds of essential oils on physical, mechanical and structural properties of chitosan films. Food Hydrocolloids 2014, 36, 287-293.

(7) Srinivasa, P.; Baskaran, R.; Ramesh, M.; Prashanth, K. H.; Tharanathan, R. Storage studies of mango packed using biodegradable chitosan film. Eur. Food Res. Technol. 2002, 215, 504-508.

(8) Koc, B.; Akyuz, L.; Cakmak, Y. S.; Sargin, I.; Salaberria, A. M.; Labidi, J.; Ilk, S.; Cekic, F. O.; Akata, I.; Kaya, M. Production and characterization of chitosan-fungal extract films. Food Biosci. 2020, 35, 100545.

(9) Kaya, M.; Mulerčikas, P.; Sargin, I.; Kazlauskaite, S.; Baublys, V.; Akyuz, B.; Bulut, E.; Tubelyte, V. Three-dimensional chitin rings from body segments of a pet diplopod species: Characterization and protein interaction studies. Mater. Sci. Eng. C 2016, 68, 716-722.

(10) Kaya, M.; Salaberria, A. M.; Mujtaba, M.; Labidi, J.; Baran, T.; Mulercikas, P.; Duman, F. An inclusive physicochemical comparison of natural and synthetic chitin films. Int. J. Biol. Macromol. 2018, 106, $1062-1070$. 
(11) Kaya, M.; Sargin, I.; Sabeckis, I.; Noreikaite, D.; Erdonmez, D.; Salaberria, A. M.; Labidi, J.; Baublys, V.; Tubelyte, V. Biological, mechanical, optical and physicochemical properties of natural chitin films obtained from the dorsal pronotum and the wing of cockroach. Carbohydr. Polym. 2017, 163, 162-169.

(12) Faria, R. R.; Guerra, R. F.; de Sousa Neto, L. R.; Motta, L. F.; de Faria Franca, E. Computational study of polymorphic structures of $\alpha$ - and $\beta$ - chitin and chitosan in aqueous solution. J. Mol. Graphics Modell. 2016, 63, 78-84.

(13) Skovstrup, S.; Hansen, S. G.; Skrydstrup, T.; Schiøtt, B. Conformational flexibility of chitosan: a molecular modeling study. Biomacromolecules 2010, 11, 3196-3207.

(14) Barragan-Fonseca, K. B.; Dicke, M.; van Loon, J. J. A. Nutritional value of the black soldier fly (Hermetia illucens L.) and its suitability as animal feed - a review. J. Insects as Food Feed 2017, 3, $105-120$.

(15) Miranda, C. D.; Cammack, J. A.; Tomberlin, J. K. Life-History Traits of the Black Soldier Fly, Hermetia illucens (L.) (Diptera: Stratiomyidae), Reared on Three Manure Types. Animals 2019, 9, 281.

(16) Diener, S.; Studt Solano, N. M.; Roa Gutiérrez, F.; Zurbrügg, C.; Tockner, K. Biological treatment of municipal organic waste using black soldier fly larvae. Waste Biomass Valorization 2011, 2, 357-363.

(17) Spranghers, T.; Ottoboni, M.; Klootwijk, C.; Ovyn, A.; Deboosere, S.; De Meulenaer, B.; Michiels, J.; Eeckhout, M.; De Clercq, P.; De Smet, S. Nutritional composition of black soldier fly (Hermetia illucens) prepupae reared on different organic waste substrates. J. Sci. Food Agric. 2017, 97, 2594-2600.

(18) Schiavone, A.; De Marco, M.; Martínez, S.; Dabbou, S.; Renna, M.; Madrid, J.; Hernandez, F.; Rotolo, L.; Costa, P.; Gai, F.; Gasco, L. Nutritional value of a partially defatted and a highly defatted black soldier fly larvae (Hermetia illucens L.) meal for broiler chickens: apparent nutrient digestibility, apparent metabolizable energy and apparent ileal amino acid digestibility. J. Anim. Sci. Biotechnol. 2017, 8, 51.

(19) Caligiani, A.; Marseglia, A.; Leni, G.; Baldassarre, S.; Maistrello, L.; Dossena, A.; Sforza, S. Composition of black soldier fly prepupae and systematic approaches for extraction and fractionation of proteins, lipids and chitin. Food Res. Int. 2018, 105, 812-820.

(20) Waśko, A.; Bulak, P.; Polak-Berecka, M.; Nowak, K.; Polakowski, C.; Bieganowski, A. The first report of the physicochemical structure of chitin isolated from Hermetia illucens. Int. J. Biol. Macromol. 2016, 92, 316-320.

(21) Purkayastha, D.; Sarkar, S. Physicochemical Structure Analysis of Chitin Extracted from Pupa Exuviae and Dead Imago of Wild Black Soldier Fly (Hermetia illucens). J. Polym. Environ. 2020, 28, 445-457.

(22) Kaya, M.; Ravikumar, P.; Ilk, S.; Mujtaba, M.; Akyuz, L.; Labidi, J.; Salaberria, A. M.; Cakmak, Y. S.; Erkul, S. K. Production and characterization of chitosan based edible films from Berberis crataegina's fruit extract and seed oil. Innovative Food Sci. Emerging Technol. 2018, 45, 287-297.

(23) Karaca, B.; Çöleri Cihan, A.; Akata, I.; Altuner, E. M. AntiBiofilm and Antimicrobial Activities of Five Edible and Medicinal Macrofungi Samples on Some Biofilm Producing Multi Drug Resistant Enterococcus Strains. Turk. J. Agric. Food Sci. Technol. 2020, 8, 69-80.

(24) Xu, Z.; Liang, Y.; Lin, S.; Chen, D.; Li, B.; Li, L.; Deng, Y. Crystal violet and XTT assays on Staphylococcus aureus biofilm quantification. Curr. Microbiol. 2016, 73, 474-482.

(25) Vestby, L. K.; Møretrø, T.; Langsrud, S.; Heir, E.; Nesse, L. L. Biofilm forming abilities of Salmonella are correlated with persistence in fish meal- and feed factories. BMC Vet. Res. 2009, 5, 20.

(26) Asahi, Y.; Noiri, Y.; Igarashi, J.; Suga, H.; Azakami, H.; Ebisu, S. Synergistic effects of antibiotics and an $\mathrm{N}$-acyl homoserine lactone analog on Porphyromonas gingivalis biofilms. J. Appl. Microbiol. 2012, 112, 404-411.

(27) Kaya, M.; Baran, T.; Mentes, A.; Asaroglu, M.; Sezen, G.; Tozak, K. O. Extraction and Characterization of $\alpha$-Chitin and
Chitosan from Six Different Aquatic Invertebrates. Food Biophys. 2014, 9, 145-157.

(28) Govindan, S.; Nivethaa, E. A. K.; Saravanan, R.; Narayanan, V.; Stephen, A. Synthesis and characterization of chitosan-silver nanocomposite. Appl. Nanosci. 2012, 2, 299-303.

(29) Bourtoom, T. Plasticizer effect on the properties of biodegradable blend film from rice starch-chitosan. Songklanakarin J. Sci. Technol. 2008, 30, 149-156.

(30) Li, Z.; Wang, X.; Zhang, X.; Yang, Y.; Duan, J. A high-efficiency and plane-enhanced chitosan film for cefotaxime adsorption compared with chitosan particles in water. Chem. Eng. J. 2020, 2020, 127494.

(31) Yang, X.; Tu, Y.; Li, L.; Shang, S.; Tao, X.-m. Well-dispersed chitosan/graphene oxide nanocomposites. ACS Appl. Mater. Interfaces 2010, 2, 1707-1713.

(32) Dou, B.; Dupont, V.; Williams, P. T.; Chen, H.; Ding, Y. Thermogravimetric kinetics of crude glycerol. Bioresour. Technol. 2009, 100, 2613-2620.

(33) Luo, X.; Hu, S.; Zhang, X.; Li, Y. Thermochemical conversion of crude glycerol to biopolyols for the production of polyurethane foams. Bioresour. Technol. 2013, 139, 323-329.

(34) Martínez-Camacho, A. P.; Cortez-Rocha, M. O.; EzquerraBrauer, J. M.; Graciano-Verdugo, A. Z.; Rodriguez-Félix, F.; CastilloOrtega, M. M.; Yépiz-Gómez, M. S.; Plascencia-Jatomea, M. Chitosan composite films: Thermal, structural, mechanical and antifungal properties. Carbohydr. Polym. 2010, 82, 305-315.

(35) Kumar, M. N. V. R. A review of chitin and chitosan applications. React. Funct. Polym. 2000, 46, 1-27.

(36) Kittur, F. S.; Harish Prashanth, K. V.; Udaya Sankar, K.; Tharanathan, R. N. Characterization of chitin, chitosan and their carboxymethyl derivatives by differential scanning calorimetry. Carbohydr. Polym. 2002, 49, 185-193.

(37) Tiğlı, R. S.; Karakeçili, A.; Gümüşderelioğlu, M. In vitro characterization of chitosan scaffolds: influence of composition and deacetylation degree. J. Mater. Sci.: Mater. Med. 2007, 18, 1665-1674.

(38) De Queiroz Antonino, R.; Lia Fook, B.; De Oliveira Lima, V.; de Farias Rached, R.; Lima, E.; Da Silva Lima, R.; Peniche Covas, C.; Lia Fook, M. Preparation and characterization of chitosan obtained from shells of shrimp (Litopenaeus vannamei Boone). Mar. Drugs 2017, 15, 141.

(39) Paulino, A. T.; Simionato, J. I.; Garcia, J. C.; Nozaki, J. Characterization of chitosan and chitin produced from silkworm crysalides. Carbohydr. Polym. 2006, 64, 98-103.

(40) Yen, M.-T.; Yang, J.-H.; Mau, J.-L. Physicochemical characterization of chitin and chitosan from crab shells. Carbohydr. Polym. 2009, 75, 15-21.

(41) Al-Naamani, L.; Dobretsov, S.; Dutta, J. Chitosan-zinc oxide nanoparticle composite coating for active food packaging applications. Innovat. Food Sci. Emerg. Technol. 2016, 38, 231-237.

(42) Rubentheren, V.; Ward, T. A.; Chee, C. Y.; Nair, P. Physical and chemical reinforcement of chitosan film using nanocrystalline cellulose and tannic acid. Cellulose 2015, 22, 2529-2541.

(43) Zheng, K.; Xiao, S.; Li, W.; Wang, W.; Chen, H.; Yang, F.; Qin, C. Chitosan-acorn starch-eugenol edible film: Physico-chemical, barrier, antimicrobial, antioxidant and structural properties. Int. J. Biol. Macromol. 2019, 135, 344-352.

(44) Liu, Y.; Wang, S.; Lan, W. Fabrication of antibacterial chitosanPVA blended film using electrospray technique for food packaging applications. Int. J. Biol. Macromol. 2018, 107, 848-854.

(45) Mollah, M. Z. I.; Akter, N.; Quader, F. B.; Sultana, S.; Khan, R. A. Biodegradable Colour Polymeric Film (Starch-Chitosan) Development: Characterization for Packaging Materials. Open J. Org. Polym. Mater. 2016, 06, 11-24.

(46) Hosokawa, J.; Nishiyama, M.; Yoshihara, K.; Kubo, T. Biodegradable film derived from chitosan and homogenized cellulose. Ind. Eng. Chem. Res. 1990, 29, 800-805.

(47) Dean, K.; Sangwan, P.; Way, C.; Zhang, X.; Martino, V. P.; Xie, F.; Halley, P. J.; Pollet, E.; Avérous, L. Glycerol plasticised chitosan: A 
study of biodegradation via carbon dioxide evolution and nuclear magnetic resonance. Polym. Degrad. Stabil. 2013, 98, 1236-1246.

(48) Gursoy, M.; Sargin, I.; Mujtaba, M.; Akyuz, B.; Ilk, S.; Akyuz, L.; Kaya, M.; Cakmak, Y. S.; Salaberria, A. M.; Labidi, J.; Erdem, N. False flax (Camelina sativa) seed oil as suitable ingredient for the enhancement of physicochemical and biological properties of chitosan films. Int. J. Biol. Macromol. 2018, 114, 1224-1232.

(49) Petrova, O. E.; Sauer, K. Sticky situations: key components that control bacterial surface attachment. J. Bacteriol. 2012, 194, 24132425.

(50) Feng, G.; Cheng, Y.; Wang, S.-Y.; Borca-Tasciuc, D. A.; Worobo, R. W.; Moraru, C. I. Bacterial attachment and biofilm formation on surfaces are reduced by small-diameter nanoscale pores: how small is small enough? npj Biofilms Microbiomes 2015, 1, 1-9.

(51) Langer, J.; Jimenez de Aberasturi, D.; Aizpurua, J.; AlvarezPuebla, R. A.; Auguié, B.; Baumberg, J. J.; Bazan, G. C.; Bell, S. E.; Boisen, A.; Brolo, A. G. Present and future of surface-enhanced Raman scattering. ACS Nano 2019, 14, 28-117.

(52) Sakir, M.; Pekdemir, S.; Karatay, A.; Küçüköz, B.; Ipekci, H. H.; Elmali, A.; Demirel, G.; Onses, M. S. Fabrication of plasmonically active substrates using engineered silver nanostructures for SERS applications. ACS Appl. Mater. Interfaces 2017, 9, 39795-39803.

(53) Barman, B.; Dhasmana, H.; Verma, A.; Kumar, A.; Chaudhary, S. P.; Jain, V. K. Formation of plasmonic silver nanoparticles using rapid thermal annealing at low temperature and study in reflectance reduction of Si surface. Adv. Nat. Sci.: Nanosci. Nanotechnol. 2017, 8, 035010 .

(54) Karabel Ocal, S.; Patarroyo, J.; Kiremitler, N. B.; Pekdemir, S.; Puntes, V. F.; Onses, M. S. Plasmonic assemblies of gold nanorods on nanoscale patterns of poly(ethylene glycol): Application in surfaceenhanced Raman spectroscopy. J. Colloid Interface Sci. 2018, 532, 449-455.

(55) Pekdemir, S.; Karabel, S.; Kiremitler, N. B.; Liu, X.; Nealey, P. F.; Onses, M. S. Modulating the Kinetics of Nanoparticle Adsorption for Simple and High-Yield Fabrication of Plasmonic Heterostructures as SERS Substrates. ChemPhysChem 2017, 18, 2114-2122.

(56) Wang, Y.; Wang, M.; Shen, L.; Sun, X.; Shi, G.; Ma, W.; Yan, X. High-performance flexible surface-enhanced Raman scattering substrates fabricated by depositing $\mathrm{Ag}$ nanoislands on the dragonfly wing. Appl. Surf. Sci. 2018, 436, 391-397.

(57) Salem, S.; Sakir, M.; Sahin, K.; Korkmaz, I.; Yavuz, E.; Sarp, G.; Onses, M. S.; Yilmaz, E. Low Bandgap Microsphere-like Magnetic Nanocomposite: An Enhanced Photocatalyst for Degradation of Organic Contaminants and Fabrication of SERS-Active Surfaces. Colloids Surf., A 2020, 589, 124436.

(58) Kiremitler, N. B.; Pekdemir, S.; Patarroyo, J.; Karabel, S.; Torun, I.; Puntes, V. F.; Onses, M. S. Assembly of plasmonic nanoparticles on nanopatterns of polymer brushes fabricated by electrospin nanolithography. ACS Macro Lett. 2017, 6, 603-608.

(59) Fukui, K. The role of frontier orbitals in chemical reactions (Nobel Lecture). Angew. Chem., Int. Ed. Engl. 1982, 21, 801-809.

(60) Fleming, I. Frontier Orbitals and Organic Chemical Reactions; Wiley, 1977. 